update was delivered to all staff utilising the resuscitation council 'lifesaver' app. As there are no hospice specific resuscitation guidelines, our was updated in line with adapted guidance for primary care and community hospitals, to incorporate the skills of rotational doctors with advanced life support certification. An emergency trolley was introduced to centralise equipment, with an added laryngeal mask airway and a pre-connected bag and mask, and a second AED was purchased to provide quick access for the whole hospice.

Conclusions There are no specific best practice guidelines for managing cardiac arrest in the hospice setting. Following a clinical incident, we have adapted the resuscitation council guidance to develop a local policy. In the future, as we care for more patients earlier in their illness, this contentious issue is likely to present more frequently. Developing a local policy could help ensure high quality resuscitation care within the hospice in response to the changing needs of palliative care patients in the future.

\section{ONE HOSPITAL TEAM, THREE SITES}

Katherine Frew, Leonie Armstrong, Carole Duff, Louise Whitfield, Hannah Hall, Patricia Robson, Jennifer Samuelson, Kay Wood, Gillian Watson, Joanna Whitchurch, Anna Office, Abbie Easton, Jeanette Doran, Emma McDougall, Hannah Gunn. Northumbria Healthcare NHS Foundation Trust, Marie Curie

\subsection{6/spcare-2020-PCC.144}

Background Northumbria Healthcare NHS FT specialist palliative care hospital liaison service (HLT) is a unified team working across three acute hospital sites with one Specialist Emergency Care Hospital (NSECH). Patients are admitted to specialty wards in NSECH for urgent assessment and treatment. If their condition is stable but requires them to stay in hospital for longer than 48 hours they are transferred to one of the other two 'base' hospitals (WGH or NTGH) for ongoing medical, and palliative, care.

Methods Data were collected using a standardised database across the three sites. Age, diagnosis, Australian modified Karnofsky Performance Scale (AKPS), phase of illness, and duration of episode of care (time from referral to discharge/death or transfer) were analysed for three sites between August 2018 August 2019.

Results Data demonstrates that patients in NSECH were younger, more likely to have cancer (66\%), and had a mean duration of episode of care of 1 day. In contrast, patients in the base hospitals were older, with $66 \%$ and $73 \%$ of patients, respectively, over 75: in NTGH $40 \%$ of patients were over 85 years old. These patients were more likely to have nonmalignant disease $(45 \%$ with non-malignant disease), and frailty was the primary diagnosis in 13\%; frailty and dementia combined were the primary diagnoses in $18 \%$. In spite of this, AKPS was similar across all three sites, with the majority of patients being $30 \%$ or less.

Discussion Acute services across the whole trust have been transformed since NSECH opened, and the HLT patient population across the three sites has radically changed. This is a responsive team which has adapted to patient need on the individual sites, and reconfigured in an iterative manner according to this need. Future palliative care services must be able to adapt and respond to the increasingly dynamic demands of the population.

\section{CAN THE SPICT BE APPLIED RETROSPECTIVELY TO IDENTIFY PATIENTS WITH UNMET SPECIALIST PALLIATIVE CARE NEEDS WHO ATTEND ED WITH BREATHLESSNESS?}

Rebecca Gardiner, Gilli Erez. London North West Thames University Healthcare Trust

\subsection{6/spcare-2020-PCC. 145}

Background There has been growing recognition that patients with the symptoms of breathlessness frequently attend the Emergency Department (ED) as they progress towards the end of life. This may represent a cohort of patients who have unidentified and unmet palliative care needs. We investigated whether the SPICT could identify these patients during an acute attendance to the ED.

Methods Retrospective data collection from electronic records on patients who attended ED in December 2018 aged over 65 and had 'breathless' equivalent in their triage. A data collection tool was created in Excel. Patient demographics, number of previous admission in the preceding 2 years, presenting complaint were collected, and then assessed against SPICT. Data on the same patients was then collected until December 2019 on further admissions, attendances and mortality. They were then re-scored against the SPICT.

Results A total of 2767 attendances in December met the criteria. The first 2 days of December were analysed. Data was collected on 16 patients. Of these, 7 patients met the SPICT criteria. In $70 \%$ admission was due to exacerbation of chronic disease. 1 year later 5 patients were still alive. On re-assessment only 4 out of 7 met the SPICT criteria. Patients who met the SPICT had a mean number of attendances to ED of 5.4, compared to 3.8. Both patients who died met the SPICT criteria.

Conclusions Our data suggests that the SPICT cannot be applied retrospectively or electronically to identify patients in the acute setting who would benefit from specialist palliative care input. Trends were that patients who did meet the SPICT had a greater number of attendances to ED. Limitations include small sample size, and reliance on correct data entry at the time of attendance. We suggest further data needs to be collected to create a tool specific to ED.

\section{BIG DATA ON EARLY IDENTIFICATION OF PATIENTS WITH PALLIATIVE CARE NEEDS: BARRIERS AND OPPORTUNITIES}

ME Gas López, MS Giménez Campos, Mateos MJ Caballero, A Duarte-Martinez, Ferrer J Garcés, Martinez B Valdivieso. Joint Research Unit in ICT applied to reengineering sociosanitary process; Departament de Salut Valencia La Fe; Joint Research Unit in ICT applied to reengineering socio-sanitary process; Polibienestar Research Institute - University of Valencia; Polibie

\subsection{6/spcare-2020-PCC.146}

Introduction The aim of this study was to explore the views of experts on the use of big data (BD) advanced analytics (i.e: machine learning, deep learning or artificial intelligence techniques) on the identification of frail older patients with nonmalignant diseases who could benefit from early palliative care (PC).

Methods This descriptive study corresponds to the first round of a Delphi study currently under performance. Participants were asked through a questionnaire survey about the level of 
agreement regarding i) the use of advanced analytics on specific clinical scenarios and ii) the critical aspects when using these applications on the frame of PC. The sample included professionals from different countries and disciplines with expertise on chronicity, geriatrics and/or PC. Consensus was defined as $>70 \%$ of subjects agreement.

Results At this time, fifteen experts have answered the questionnaire $(37.5 \%$ of whom invited to participate). $78.6 \%$ were women, $71.4 \%$ were working in a clinical setting $>15$ years as physicians (57.1\%), nurses (35.7\%) and psychologists (7.1\%) $80 \%$ of them considered that the strategy based on an automatic tool (BD based) combined with front-line healthcare staff is the best way for identification of patients who could benefit from early PC approach. They assessed as 'very' and 'extremely useful' the use of BD models on research applications, both as a population health management tool (clinical clustering) and as a tool to improve the prediction of an outcome risk. However, the need for clinical validation and the dearth of evidence of practical benefits are the main critical aspects on the implementation of these tools.

Conclusions The view of experts can contribute to guide BD applications on advanced stages of illnesses. The consensus about the opportunities and gaps on the implementation of these tools will support clinicians in decision making processes.

\section{EVALUATION OF THE PROCESS OF SENDING OUT OACC QUESTIONNAIRE TO PATIENTS, PRIOR TO THEIR FIRST PALLIATIVE CARE NURSE SPECIALIST VISIT/ APPOINTMENT, JUNE 2019}

Julie Gater, Davina Bell, Emer McKenna. Douglas Macmillan Hospice

10.1136/spcare-2020-PCC. 147

The OACC Assessment Questionnaire is sent out to all appropriate patients at telephone triage who are assessed as needing to be seen within 1-2 Weeks. The Questionnaire looks at IPOS as part of OACC.

This process allows for the Patients to have more time to review their symptoms and to think about their main problems of concern over the last week, along with any practical problems resulting from their illness, prior to their Palliative Care Nurse Specialist visiting them in their own home for their first holistic assessment.

By enabling an opportunity for the Patients to complete the Questionnaire themselves, it reduces reliance on Healthcare Professional Staff assisting them, and gives more autonomy/direction about key issues for them. It can also be used as a channel for Patients and Families to have some open conversation prior to their first visit/assessment, and to give insight into areas we will cover, which are different to a District Nursing assessment.

This review aimed to establish the benefit of sending out the Questionnaire. Also to examine if the action of recording data within the process was robust, and to identify any areas that could be improved.

A random sample of 34 patients was selected for retrospective review of activity from sending out the Questionnaire to examination whether this was completed by the patient or helped by the nurse to complete on the first visit, and scrutiny of the data recorded on Crosscare thereafter.
Overall, it was found that there was benefit of sending out the OACC Questionnaires with 50\% of Patients completing this prior their PCNS visit. However, following analysis of the data recorded, we established that we needed to revisit training, particularly for PCNSs for navigating the IPOS windows and recording data therein.

\section{PALLIATIVE CARE FOR HOMELESS PEOPLE: A SYSTEMATIC REVIEW OF SYSTEMATIC REVIEWS FOR AN EVIDENCE BASED CLINICAL GUIDELINE}

Anna Gray. King's College London and St Francis Hospice (Romford)

\subsection{6/spcare-2020-PCC.148}

Background Homeless people die much younger and have higher rates of ill-health compared to the general population. This systematic review of systematic reviews seeks to understand 1. views and preferences of homeless people regarding palliative care, 2. barriers and facilitators to homeless people accessing palliative care and 3. evidence for advance care planning interventions in palliative care for homeless people. This informed the development of a guideline for use by healthcare professionals providing palliative care for homeless patients.

Methods Searches took place of PubMed, Embase, PscyhINFO, CINAHL, Web of Science, Medline and Cochrane Library from inception to December 2019. The quality of the studies was assessed using SIGN Methodology checklist 1. The GRADE system was then used to assess the quality of the evidence and strength of the recommendations. Themes were identified following data analysis.

Results Five systematic reviews met the eligibility criteria. Three were rated as moderate quality, one as poor quality and one as unacceptable quality. Nine types of themes were then identified. First, views and preferences; homeless people recall poor experiences of healthcare in the past and have unique concerns regarding their health. Five barriers to palliative care were also identified, namely; lack of basic needs being met, having competing priorities, being ill-informed about their condition, hospice and hostel staff having insufficient knowledge, and poor co-ordination of care. The emerging facilitator was that homeless patients trust the staff they already know. Finally, many homeless patients benefit from advance care planning interventions, if they feel able to engage in the process. The quality of the recommendations was moderate to low. The strength of the recommendations was mostly strong.

Conclusions In this review, the views, preferences, barriers, facilitators, and interventions in palliative care for homeless people were established, informing a new clinical guideline.

\section{IMPLEMENTATION OF TREATMENT ESCALATION PLANS (TEP) AND DNACPR DECISIONS AT ST GEORGE'S UNIVERSITY HOSPITALS NHS FOUNDATION TRUST}

Amy Hawkins, Megan L Papworth. St George's University Hospitals NHS Foundation Trust

\subsection{6/spcare-2020-PCC. 149}

Introduction Treatment escalation plans (TEP) are a tool to promote advance care planning, appropriate decision making and good communication between professionals, patients and 